\title{
Minitrack Introduction for Social Media Management in Big Data Era
}

\author{
Xiangbin Yan \\ xbyan@ustb.edu.cn
}

\author{
Mingxin Gan \\ ganmx@ustb.edu.cn
}

\author{
Hua (Jonathan) Ye \\ jonathan.ye@uwaterloo.ca
}

\begin{abstract}
Papers in this minitrack cover several interesting topics regarding social media management in big data era. The minitrack presents six papers in total. Topics of these papers include novel social media tools, techniques and methods in social media management, opinion formation in social media, content commercialization, personalized social media recommendation, customer preference analysis in social media, co-branding via social media, particularly in regard to community and individual relationship. Such papers enrich and provide multidisciplinary inspirations to extant research in the field of social media management.
\end{abstract}

\section{Opinion Formation in Social Media}

This study focuses on investigating how individuals form opinions from passive social media information consumption. It considers the combinant role of three types of social media information i.e., image, video and text message in an individual's opinion formation. It then proposes a method to determine the threshold that various combinations of different type of social media information can help the population form opinions.

\section{Customer Preference Analysis in Social Media}

A study investigates the effects of perceived interactivity of virtual brand community on customer perceived value as well as on brand preference. It differentiates the interactivity regarding with the community and customers and hypothesizes their impacts on perceived value and brand preferences. This study interestingly finds that interactivity can enhance perceived value and brand preference.

\section{Personalized Recommendation in Social Media}

Unlike most user-based music recommendation, this study develops a two-side value-based approach to artist recommendation in a streaming music scenario. It integrates both music provides and consumers to improve recommendation performance for potential listeners. It also demonstrated the performance of their method by comparing with the collaborative filtering method.

\section{Co-branding via Social Media}

This study examines the cobranding activity on social media platforms via a case study. It studies the cobranding on Instagram involving the soccer club Manchester United and the soccer player Zlatan Ibrahimović. Sentiment and emotional tone analysis are used to assess the interactions between the audience and illustrated non-verbal communication used by social media users. It also illustrates the difficulties associated with co-branding between personal and corporate brands as well asynchronous communication.

\section{Commercializing Social Media Content}

This study investigates a new business model that has been adopted by Weibo.com, i.e., paid Q\&A. Users who have expertise in certain areas can charge other users for answering their questions. Using signaling theory, this study investigates what affects others to pay to view answers from paid Q\&A from three distinct types of signals. This study interestingly explores how this new business model works and paves the way for future research. 


\section{New Tools, Techniques and Methodologies for Social Media Management}

This study introduces a new method to extract more accurate geographic and event-related information from social media. It proposes a network filtering method that uses social network ties to infer the location of social media users in a geographic community and collects data from a network of these users during a crisis. This method can receive more situational reports collected in real-time compared to other methods, supporting the formation of situational awareness of incident occurrence across a community. 